\title{
Changes in oral trace gas concentrations following orthognathic surgery and intermaxillary fixation: a case study using selected ion flow tube mass spectrometry
}

\author{
Brian M. Ross*
}

Division of Medical Sciences at the Northern Ontario School of Medicine in Thunder Bay, Ontario, MS 3002, Canada

Orthognathic surgery is frequently accompanied by intermaxillary fixation. Intermaxillary fixation impedes the maintenance of effective oral hygiene and prolonged fixation can result in periodontal disease. A potential shorter term effect is the generation of oral malodour. It is unclear, however, as to how the production of malodorous compounds in the oral cavity is altered post-surgery. Oral air concentration of sulphur containing compounds, short chain organic acids, ammonia, isoprene and acetone were measured using selected ion flow tube-mass spectrometry in a patient who had undergone orthognathic surgery with subsequent intermaxillary fixation. Total sulphide levels rose approximately 5 -fold during fixation with metal ties, with smaller increases recorded for the other compounds measured with the exception of isoprene which remained close to baseline levels. Organic acid levels declined markedly once elastic ties had replaced metal ties, with a lesser reduction being observed in sulphide levels, with both declining further after the commencement of a chlorhexidinecontaining mouthwash. These data suggest that bacterial generation of a variety of malodorous compounds increases markedly following intermaxillary fixation. This single case also suggests that the use of elastic ties and effective oral hygiene techniques, including the use of chlorhexidine mouthwash, may help ameliorate such post-surgical effects.

Keywords: oral malodour; halitosis; oral hygiene; breath analysis; organic acids; sulphides; mass spectrometry

International Journal of Oral Science (2011) 3: 160-164. doi: 10.4248/IJOS11058

Introduction

Orthognathic surgery is a common class of procedures which aim to correct conditions of the face and jaw including temperomandibular joint dysfunction, sleep apnea, and a variety of craniofacial abnormalities such as orthodontic problems [1]. The procedure usually involves

*Correspondence: Brian M. Ross

Tel: 001807766 7394; Fax: 0018077667370

E-mail: brian.ross@nosm.ca

Received 4 September 2010; Accepted 22 January 2011 osteotomy of the bones of the maxilla and/or mandible to allow repositioning into the required alignment. Following surgery intermaxillary fixation is frequently performed by means of wires attached to brackets on the teeth. This "wiring shut" requires that the patient adheres to a liquid diet, and restricts or prevents their engaging in normal oral hygiene procedures such as brushing and flossing. Indeed, an increase in bacterially caused periodontal disease has been noted following intermaxillary fixation used in the treatment of jaw fractures or obesity [2-4].

A useful indicator of oral hygiene is the bacteria- 
derived volatiles produced in the oral cavity [5]. Specifically, bacteria present in the mouth, in particular the dorsal surface of the tongue, catabolise food stuffs to produce a variety of chemicals, some of which result in little odour at typical concentrations, such as ammonia [6], while others are a source of malodour [5]. The predominant odiferous compounds derive from sulphur-containing amino acids and include hydrogen sulphide $\left(\mathrm{H}_{2} \mathrm{~S}\right)$, methylmercaptan $\left(\mathrm{CH}_{3} \mathrm{SH}\right)$, dimethylsulphide $\left(\mathrm{CH}_{3} \mathrm{SCH}_{3}\right)$, and dimethyldisulphide $\left(\mathrm{CH}_{3} \mathrm{~S}_{2} \mathrm{CH}_{3}\right)$, although additional compounds such as short-chain organic acids (butyric, propionic and valeric), polyamines and indole may also contribute [5]. Measurement of such compounds in the oral cavity may offer a means to monitor oral hygiene following surgery and intermaxillary fixation. Quantification of such chemically diverse gases, normally present in the low to high parts per billion by volume range in the oral cavity is, however, difficult using conventional means such as gas chromatography, requiring multiple time consuming chromatographic separations. A new technique, selected ion flow tube-mass spectrometry (SIFT-MS), can readily achieve this task and provide a more complete picture of what is occurring in the mouth. SIFT-MS is a form of chemical ionisation mass spectrometry in which trace gases are reacted with ions, such as $\mathrm{H}_{3} \mathrm{O}^{+}, \mathrm{NO}^{+}$and $\mathrm{O}_{2}^{+}$to form charged product ions at a known rate thereby allowing real time absolute quantification of chemically diverse trace gases [7]. For example, SIFT-MS can readily assay sulphides, ammonia and organic acids simultaneously, a capability not matched by conventional chromatographic separations. SIFT-MS is related to other ionisation mass spectrometry techniques such proton transfer-mass spectromety (PTR-MS) and atmospheric ionisation-mass spectroscopy (API-MS) which can also be used to analyse human breath [8]. SIFT-MS has the advantage, however, of being able to use multiple precursor ions to differentiate isomeric and isobaric compounds hence generally increasing the chemical resolution of the technique over PTR- and API-MS [7]. The current limits of detection of the technique are in the low PPBV and high PPTV ranges. This is sufficient to allow the quantification of the known odiferous oral volatiles using previously reported mass spectrometric data [7, 9]. SIFT-MS was therefore chosen to investigate oral gases in a patient who had recently undergone orthognathic surgery.

\section{Methods}

The patient was a 42 year old male who had undergone bilateral sagital split osteotomy and advancement of the mandible along with genioplasty. Post-surgery the mandibles were held in place by means of an acrylic splint and intermaxillary fixation using metal ties. He had received intravenous dextramethasone and cephalexin during his two day hospital stay, and continued taking cephalexin for 5 days after leaving the hospital. Up until the cessation of continuous intermaxillary fixation the patient consumed a liquid diet consisting mainly of creamed soups and liquid "meal replacements". There was no history of peridontal disease or oral malodour.

Using an a protocol approved by the Local Ethics Board of Lakehead University, at seven and eight days prior to surgery baseline oral air measurements were taken around 9:00 am. The patient had refrained from eating past midnight the previous evening and had not used any mouthwash or rinse since the previous evening. The subject was asked to nose breathe for 3 minutes with subsequent sampling of oral air for 45 seconds via a $5 \mathrm{~cm}$ PTFE tube with outer diameter of $1 / 16$ inch at a flow rate of approximately 0.2 standard $\mathrm{cm}^{3} \cdot \mathrm{s}^{-1}$. In previous work the tubing had been placed approximately $2.5 \mathrm{~cm}$ within the mouth superior to the tongue [10]. In this study, however, the wearing of a splint prevented this and therefore the tube was inserted through the lips to just reach the teeth. The other end of the tube was connected to the 6 foot long PTFE sampling line of a Profile-3 SIFT-MS (Instrument Science, UK). The tube had an outer diameter of 1/16 inch and an inner diameter of $0.025 \mathrm{inch}$, and was held at a temperature of $100{ }^{\circ} \mathrm{C}$. The sampling flow was reduced to the desired rate using a short length of 1/16 inch PTFE tubing which possessed an internal diameter of 0.007 inch [9]. The compounds of interest (sulphides, organic acids, ammonia, isoprene and acetone) were quantified by monitoring the specific ion product of the reaction between the compound of interest and the precursor ion transformed into absolute concentrations using a predetermined reaction rate coefficient [6-7, 11-14]. For each compound the parameters were (in order compound : precursor, product ions, reaction rate constant in units of $\mathrm{cm}^{3} \cdot \mathrm{s}^{-1} \times 10^{-9}$ ) - hydrogen sulphide: $\mathrm{H}_{3} \mathrm{O}^{+}, \mathrm{m} / z 35$ and 53, 1.6; methylmercaptan: $\mathrm{H}_{3} \mathrm{O}^{+}, m / z$ 49, 67 and 85, 2.5; dimethyl disulphide: $\mathrm{NO}^{+}$, $m / z$ 94, 2.4; dimethyl disulphide: $\mathrm{O}_{2}{ }^{+}, m / z 62$ (with multiplier of 1.6), 2.2; isoprene: $\mathrm{H}_{3} \mathrm{O}^{+}, \mathrm{m} / z$ 69, 1.6; acetone: $\mathrm{H}_{3} \mathrm{O}^{+}, \mathrm{m} / z 59$ and 77, 3.9; ammonia: $\mathrm{H}_{3} \mathrm{O}^{+}, \mathrm{m} / z$ 18, 36 and 54, 2.6; acetic acid: $\mathrm{NO}^{+}, m / z 90$ and 108, 1.2; propionic acid: $\mathrm{H}_{3} \mathrm{O}^{+}, 75,93$ and 111 (with correction for isotopologue effect of the tri-hydrate of water $\mathrm{m} / \mathrm{z}$ 73), 2.7; butyric acid: $\mathrm{NO}^{+}, m / z \quad 71$ and 118, 1.9; and valeric acid: $\mathrm{NO}^{+}, m / z 85$ and 132, 2.4. Precursor ion count rates for all ions exceeded $800000 \mathrm{cps}$, with precursor ion purity exceeding $98.5 \%$. Precursor and product ions were monitored sequentially with precursor 
ion being counted for 0.1 second each cycle and product ion for 0.5 seconds. Depending on the number of ions counted per cycle, this resulted in data points being collected approximately every $3-5$ seconds and averaged over the sampling period. It should be noted that SIFTMS is vulnerable to the presence of isobaric compounds and isotopologues which give rise to a product possessing the ions with the same $\mathrm{m} / \mathrm{z}$ as the compound of interest. By examining the current SIFT-MS spectra library and considering which compounds are likely to be present in oral air, the above ionic products are likely to derive from the described compounds, and the remainder of this paper making this assumption. Having said this it should be noted that the monohydrate of the reaction between propionic acid and $\mathrm{H}_{3} \mathrm{O}^{+}$is isobaric with that of the reaction with toluene, the presence of which could interfere with the measurement of the acid [15].

\section{Results and Discussion}

The baseline measurements (Table 1) demonstrated low sulphur compound levels (total sulphur compound concentration was $37 \mathrm{PPBV}$ as illustrated in the Figure 1) consistent with having no history of oral malodour [16].
The other compounds measured were negligible when compared to other published studies [6-7, 11-14], although this, to the best of the authors knowledge, is the first report describing oral air concentrations of propionic and valeric acid. Baseline concentrations of the sulphides, ammonia, isoprene and acetone could be easily differentiated from ambient values, although, of the organic acids only acetic acid was above ambient concentrations.

Thirteen days following surgery, the mandibles remained immobilised by means of an acrylic splint and wired intermaxillary fixation. The patient had been lightly brushing the facial surface of his teeth, avoiding the surgical incisions, and using a menthol-based mouthwash, the latter being used to give temporary relief to a reported tongue irritation. He also stated that his spouse had complained that he had developed oral malodour. Total sulphur levels had increased approximately 5-fold at post-surgery days 13 and 14, having an absolute value of 186 PPBV with hydrogen sulphide showing the largest increase (Figure 1). Such concentrations are consistent with the reported subjective impression of malodour [16]. Ammonia and acetone levels were also increased at this time point, while isoprene concentrations were slightly lower (Table 1). The oral concentrations of all four organic acids assayed (Table 1) were also increased, in

Table 1 Oral air chemical concentrations measured using SIFT-MS following orthognathic surgery

\begin{tabular}{|c|c|c|c|c|c|c|c|c|c|}
\hline \multirow{2}{*}{ Compound } & \multirow{2}{*}{$\begin{array}{l}\text { Ambient } \\
\text { (PPBV) }\end{array}$} & \multirow{2}{*}{$\begin{array}{l}\text { Baseline } \\
\text { (PPBV) }\end{array}$} & \multicolumn{7}{|c|}{ Time after surgery (days) } \\
\hline & & & 13 & 14 & 16 & 21 & 22 & 37 & 43 \\
\hline Hydrogen sulphide & $1 \pm 1$ & 15 & $693 \%$ & $560 \%$ & $380 \%$ & $193 \%$ & $173 \%$ & $93 \%$ & $133 \%$ \\
\hline Methylmercaptan & $1 \pm 0.5$ & 9 & $367 \%$ & $422 \%$ & $278 \%$ & $200 \%$ & $133 \%$ & $78 \%$ & $67 \%$ \\
\hline Dimethyl sulphide & $0.5 \pm 1$ & 6 & $233 \%$ & $300 \%$ & $217 \%$ & $183 \%$ & $200 \%$ & $83 \%$ & $83 \%$ \\
\hline Dimethyl disulphide & $1 \pm 1$ & 4 & $525 \%$ & $550 \%$ & $325 \%$ & $125 \%$ & $75 \%$ & $150 \%$ & $125 \%$ \\
\hline Acetic acid & $12 \pm 2$ & 36 & $222 \%$ & $192 \%$ & $153 \%$ & $122 \%$ & $108 \%$ & $83 \%$ & $75 \%$ \\
\hline Propionic acid & $2 \pm 1$ & 5 & $200 \%$ & $220 \%$ & $160 \%$ & $100 \%$ & $80 \%$ & $60 \%$ & $100 \%$ \\
\hline Butyric acid & $5 \pm 2$ & 7 & $300 \%$ & $314 \%$ & $143 \%$ & $114 \%$ & $114 \%$ & $114 \%$ & $129 \%$ \\
\hline Valeric acid & $2 \pm 1$ & 6 & $200 \%$ & $233 \%$ & $67 \%$ & $117 \%$ & $83 \%$ & $67 \%$ & $67 \%$ \\
\hline Isoprene & $10 \pm 2$ & 98 & $81 \%$ & $113 \%$ & $122 \%$ & $84 \%$ & $61 \%$ & $115 \%$ & $117 \%$ \\
\hline Acetone & $12 \pm 4$ & 423 & $293 \%$ & $244 \%$ & $312 \%$ & $233 \%$ & $108 \%$ & $74 \%$ & $121 \%$ \\
\hline Ammonia & $98 \pm 52$ & 875 & $217 \%$ & $208 \%$ & $153 \%$ & $126 \%$ & $94 \%$ & $62 \%$ & $72 \%$ \\
\hline
\end{tabular}

Air was sampled from the oral cavity via a PTFE tube at a rate of approximately $0.2 \mathrm{~mL} \cdot \mathrm{min}^{-1}$ for 45 seconds per measurement. The subject had nose-breathed for three minutes prior to sampling. Baseline (pre-surgery concentration) is the mean of measurements taken 7 and 8 days prior to surgery. Baseline measurement are shown as absolute concentrations whereas post-surgery concentrations are shown as a percentage relative to baseline. Ambient air measurements were made in triplicate at each time point. Since these did not differ significantly between measurement days (one way ANOVA; $P>0.05$ ) a mean value $( \pm \mathrm{SD})$ is shown. The metal ties were replaced with elastic ties after the measurement on day 14, between post-surgery days 16 and 21 the patient began to use a chlorhexidine containing mouthwash, and the splint and ties were restricted to night-time use on day 35 . 
particular that of acetic and butyric acids, confirming and extending a previous finding of increased oral acetic acid levels in a patient with oral malodour [10]. Such data provide evidence that elevated organic acid levels may play a role in oral malodour. Having said that, it is unknown as to the contribution to subjectively detected oral malodour that these absolute levels of vapour phase organic acid actually play, but which techniques such as SIFT-MS are ideally suited to investigate.

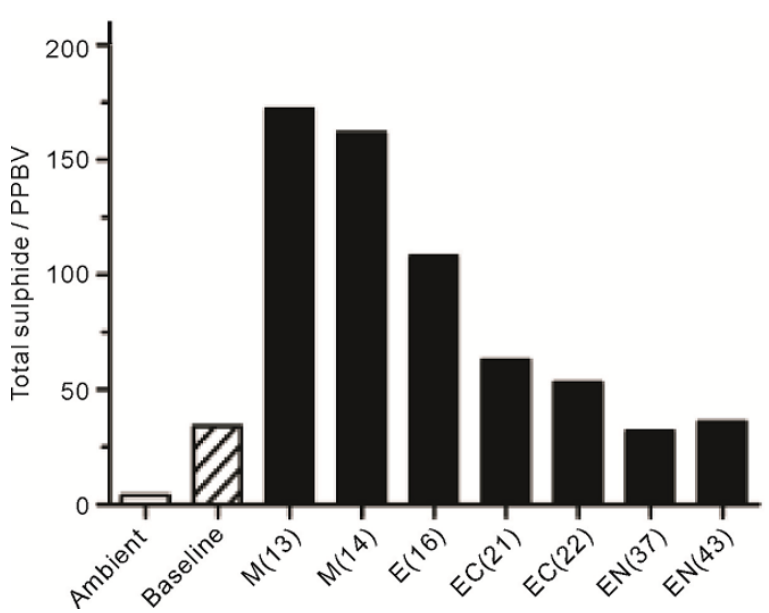

Figure 1 Sulphide concentration of oral air following orthognathic surgery and intermaxiallary fixation. Total oral sulphides, comprising the sum of hydrogen sulphide, methylmercaptan, dimethyl sulphide and dimethyl disulphide, were measured by SIFT-MS in a single patient before and after orthognathic surgery (baseline) as described in the legend to the Table. The conditions at each time point were: $M$ - intermaxillary metal ties, $E$ elastic ties, C - chlorhexidine containing mouthwash was being used, $\mathrm{N}$ - splint and ties were restricted to night-time use only. The number of days post-surgery is shown in parenthesis.

Following the measurement on day 14, the wires were replaced by elastic ties and further oral air analysis was conducted on day 16 . The use of elastics resulted in the patient being able to brush the occlusal and incisal surfaces of the teeth. At this time point the oral concentrations of ammonia had fallen (Table 1), as had sulphur containing compounds which decreased approximately 3 -fold over baseline concentrations (Figure 1). Organic acid levels remained elevated, in particular butyric acid, although they had fallen markedly from that on day 14, much more so in relative terms than that of the sulphides suggesting a qualitative difference between the processes leading to the formation of each chemical class. Oral acetone concentrations remained high, however, while isoprene concentration again showed no marked change (Table 1). These findings are not unexpected given that sulphur compounds and ammonia are produced predominantly within the mouth by microbes, while acetone and isoprene have a mainly systemic source, the former being formed in beta-oxidation of fatty acids, the latter in cholesterol biosynthesis [6]. As such elevated acetone concentrations are likely due to calorific restriction following surgery due to the need to consume a liquid diet, a conclusion supported by the fact that the patient had lost $18 \mathrm{lbs}$ in weight by 21 days post surgery. Indeed oral acetone levels remained elevated until 22 days postsurgery (Table 1), and did not fall in concert with those of ammonia and the sulphides. Furthermore, normal isoprene levels suggested that the rate of cholesterol synthesis had not changed. It should be noted, however, that the $\mathrm{m} / z 69$ product ion deriving from isoprene could also be produced by the dihydrate of the reaction between methanol and $\mathrm{H}_{3} \mathrm{O}^{+}$[17], a possibility that should be considered in future studies.

Between days 16 and 21 the patient changed their mouthwash to one containing chlorhexidine which he used twice daily. Total sulphur compound concentration remained elevated by approximately 1.7 fold on day 21 , but had markedly declined from day 16 (Figure 1). The patient's spouse had also ceased to mention the presence of oral malodour. Indeed, ammonia and dimethyl disulphide levels had returned to baseline levels by day 22 (Table 1). While these reductions may have been due to the use of the chlorhexidine containing mouthwash, which appear to more effectively counter oral malodour compared to essential oil containing mouthwashes [16], the cumulative effects of improved tooth brushing may also have played a role. On day 34 the splint and elastic ties were removed during the day and retained for night time use only, allowing the patient to return to their normal dental hygiene with a cessation of the use of the chlorhexidine-containing mouthwash. The final two oral air measurement on days 37 and 43 post-surgery show gas concentrations for all measured compounds returning to approximately baseline values (Table 1 and Figure 1).

\section{Conclusions}

To the best of the author's knowledge, this is the first investigation of oral trace gas levels following orthognathic surgery. In summary, this case study demonstrates that orally generated ammonia and malodourous sulphur containing compounds can rise markedly following orthognathic surgery combined with intermaxillary fixa- 
tion, leading to the occurrence of oral malodour, highlighting the need to consider the most effective means to lessen the impact of such procedures upon oral hygiene. The data from this single case suggests that the use of elastic ties and a chlorhexidine containing mouthwash may diminish the negative impact of the surgery. Further investigations using clinical trial type designs will be required to investigate the generalisability of these findings. The study also demonstrates the utility of SIFT-MS in the analysis of oral air, in particular the ability of the technique to quickly measure the concentrations of dissimilar chemical classes.

\section{References}

1 Reyneke JP. Essentials of orthognathic surgery. Chicago: Quintessence Publishing Co., Inc., 2003.

2 Shepard BC, Townsend GC, Goss AN. The oral effects of prolonged intermaxillary fixation by interdental eyelet wiring. Int J Oral Surg 1982; 11: 292-298.

3 Lello JL, Lello GE. The effect of interdental continuous loop wire splinting and intermaxillary fixation on the marginal gingival. Int J Oral Maxillofac Surg 1988; 17: 249-252.

4 Thor A, Andersson L. Interdental wiring in jaw fractures: effects of teeth and surrounding tissues after a one-year follow-up. Br J Oral Maxillofac Surg 2001; 39: 398-401.

5 Porter SR, Scully C. Oral malodour (halitosis). BMJ 2006; 333: 632-635.

6 Wang T, Pysanenko A, Dryahina K, Španěl P, Smith D. Analysis of breath, exhaled via the mouth and nose, and the air in the oral cavity. J Breath Res 2008; 2: 03701.

7 Španěl P, Smith D. Selected ion flow tube mass spectrometry for on-line trace gas analysis in biology and medicine. Eur J Mass Spectrom 2007; 13: 77-82.

8 Martínez-Lozano P, Fernández de la Mora J. Direct analysis of fatty acid vapors in breath by electrospray ionization and atmospheric pressure ionization-mass spectrometry. Anal
Chem 2008; 80: 8210-8215.

9 Ross BM, Babay S, Ladouceur C. The use of selected ion flow tube mass spectrometry to detect and quantify polyamines in headspace gas and oral air. Rapid Commun Mass Spectrom 2009; 23: 3973-3982.

10 Ross BM, Dadgostar N, Bloom M, McKeown L. The analysis of oral air using selected ion flow tube mass spectrometry in persons with and without a history of oral malodour. Int J Dent Hyg 2009; 7: 136-143.

11 Španěl P, Smith D. SIFT studies of the reactions of $\mathrm{H}_{3} \mathrm{O}^{+}$, $\mathrm{NO}^{+}$and $\mathrm{O}_{2}^{+}$with a series of volatile carboxylic acids and esthers. Int J Mass Spectrom Ion Processes 1998; 172: 137147.

12 Španěl P, Dryahina K, Smith D. Acetone, ammonia and hydrogen cyanide in exhaled breath of several volunteers aged 4-83 years. J Breath Res 2007; 1: 11001.

13 Pysanenko A, Španěl P, Smith D. Analysis of the isobaric compounds propanol, acetic acid and methyl formate in humid air and breath by selected ion flow tube mass spectrometry, SIFT-MS. Int J Mass Spectrom 1998; 285: $42-48$.

14 Pysanenko P, Španěl P, Smith D. A study of sulfurcontaining compounds in mouth- and nose-exhaled breath and in the oral cavity using selected ion flow tube mass spectrometry. J Breath Res 2008; 2: 46004.

15 Španěl P, Smith D. Selected ion flow tube studies of the reactions of $\mathrm{H}_{3} \mathrm{O}^{+}, \mathrm{NO}^{+}$and $\mathrm{O}_{2}^{+}$with several aromatic and aliphatic hydrocarbons. Int J Mass Spectrom 1998; 181: 110.

16 Rosenberg M, Kulkarni GV, Bosy A, McCulloch CA. Reproducibility and sensitivity of oral malodor measurements with a portable sulphide monitor. J Dent Res 1991; 70: 1436-1440.

17 Španěl P, Smith D. SIFT studies of the reactions of $\mathrm{H}_{3} \mathrm{O}^{+}$, $\mathrm{NO}^{+}$and $\mathrm{O}^{2+}$ with a series of alcohols. Int J Mass Spectrom Ion Processes 1997; 167/168: 1-12. 